\section{Uncovering the molecular conformations and adsorption free energies of aspartate at calcite growth sites}

\section{ALEXANDER BROAD}

University College London

Presenting Author: a.broad.17@ucl.ac.uk

Additives play a crucial role in biomineralisation, driving changes to the processes of crystallisation from their molecular effects on growth, to their occlusion and resulting modification of the on morphology and mechanical properties of biogenic crystals. Yet the molecular mechanisms through which they interact with growth sites and alter the crystallisation process are poorly understood. Adsorption free energies of additives can be determined experimentally by fitting Langmuir adsorption isotherms to data on fractional inhibition. [1] Where such measurements offer a macroscopic picture, only simulations are able to achieve the resolutions necessary to elucidate the molecular processes behind experimental findings. However, reproducing the conditions to derive these adsorption free energies in simulations is a complex process. This is partly due to the number of possible growth sites and molecular orientations, but also due to the challenges of fitting accurate forcefields for such mixed systems. Here, a set of force fields explicitly fit to solvation free energies [2] is used to perform a comprehensive study of aspartate at calcite growth sites.

The stability of the numerous possible conformations of the aspartate molecule at the calcite kink are investigated by choosing the several of the most promising, and running long molecular dynamics simulations. The metastable conformations identified from these initial simulations are further investigated with metadynamics with and without explicit dehydration of the kink site. These simulations are used to determine adsorption free energies which are then compared directly to experimental results. The calculated adsorption free energy shows a remarkable agreement with experimental values. This justifies the use of the chosen force fields, and offers a unique insight into the dynamics of aspartate at calcite sites. This also offers a wider insight into how carboxyl groups interact with calcite and affect its growth. The ease with which these methods can be carried out may also offer a new means through which additives can be studied extensively through collaborations between theory and experiment.

\section{References:}

[1] Montanari, Lakshtanov, Tobler, Dideriksen, Dalby, Bovet, \& Stipp (2016) Cryst. Growth Des., 16, 4813-4821

[2] Raiteri, Demichelis, Gale, Kellermeier, Gebauer, Quigley, Wright \& Walsh (2012), Faraday Discuss., 159, 61-85
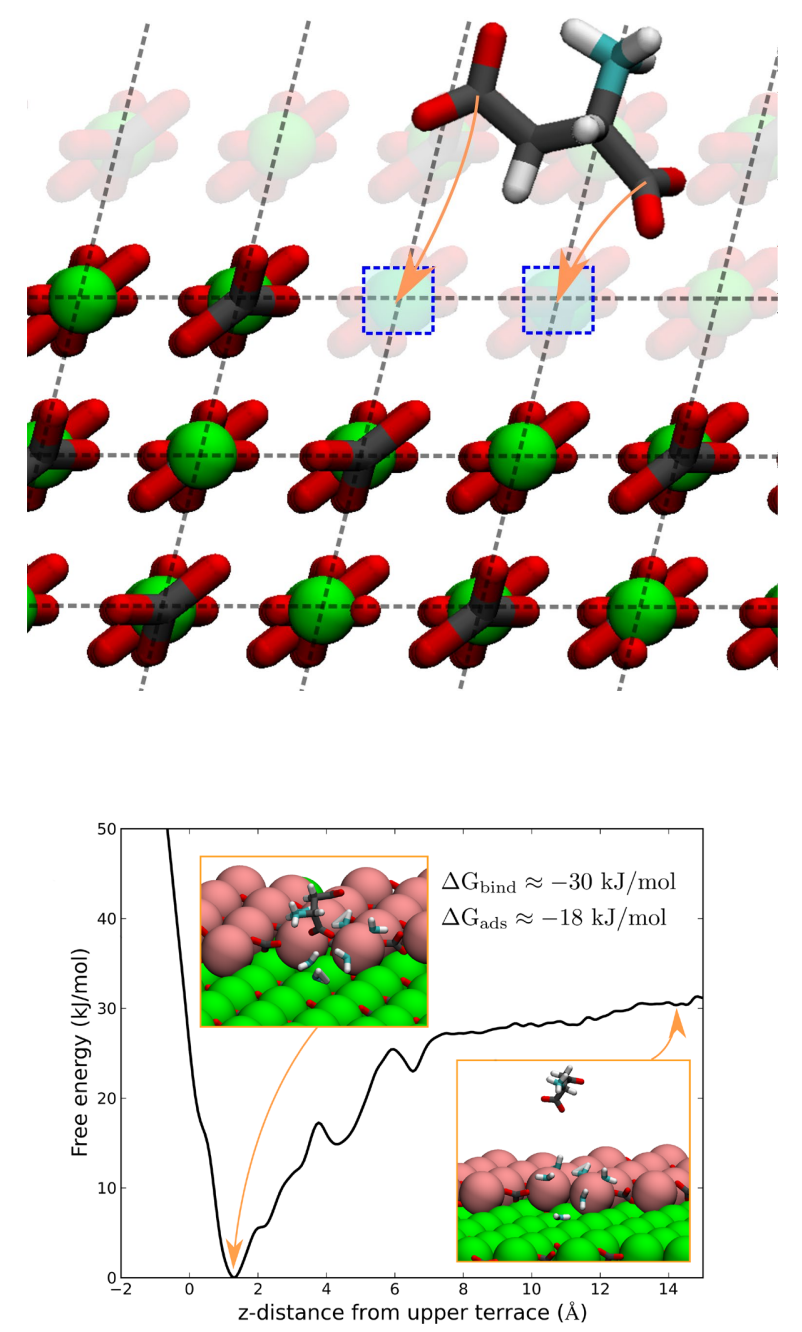